\title{
Structural, fluoride release, and 3D interfacial adhesion analysis of bioactive endodontic sealers
}

\author{
Yara Khalid ALMAIMOUNI' ${ }^{1}$ Shorouq Khalid HAMID², Kanwal ILYAS ${ }^{3}$, Asma Tufail SHAH ${ }^{3}$, Abdul MAJEED ${ }^{1}$ \\ and Abdul Samad KHAN ${ }^{1}$ \\ ${ }^{1}$ Department of Restorative Dental Sciences, College of Dentistry, Imam Abdulrahman Bin Faisal University, Dammam 31441 , Saudi Arabia \\ ${ }^{2}$ College of Dentistry, Imam Abdulrahman Bin Faisal University, Dammam 31441, Saudi Arabia \\ ${ }^{3}$ Interdisciplinary Research Center in Biomedical Materials, COMSATS University Islamabad, Lahore Campus, Lahore 54000, Pakistan \\ Corresponding author, Abdul Samad KHAN; E-mail: akhan@iau.edu.sa
}

\begin{abstract}
The experimental bioactive sealers were synthesized by incorporating fluoridated-nano-bioactive glass (F-nBG; 2.5 and 5 wt\%) in AH Plus $^{\circledR}$ (Dentsply DeTrey, Konstanz, Germany) sealer and denoted as AH-FBG2.5 and AH-FBG5, respectively. Structural pattern, setting time, flowability, and water sorption analysis were performed. The fluoride release behavior was evaluated periodically over the course of 40 days using inductively coupled plasma optical emission spectroscopy. For sealing ability, post-extraction single-rooted teeth were obturated with sealers. The percentage of voids and sealing ability were evaluated periodically using micro-computed tomography (micro-CT) followed by push-out bond strength. The Fourier transform infrared spectra showed a change in peak height with an increase in the concentration of fillers. The setting time, flowability, and water sorption of experimental groups were within the acceptable clinical range. The fluoride release, sealing ability, and bond strength of experimental sealers were significantly high. The experimental sealers have potential to overcome sealing ability issues of sealers.
\end{abstract}

Keywords: Endodontic sealers, Bioactive glass, Fluoride release, Sealibility, micro-CT

\section{INTRODUCTION}

Root canal treatment (RCT) is employed to eliminate etiological factors through chemo-mechanical methods by sealing canals to avoid recontamination ${ }^{1)}$. During obturation, sealers are essential to fill the gaps between root dentin and the filling material ${ }^{2}$. Sealers have three main functions: bonding simultaneously to root dentin and restoring materials, sealing voids and multiple foramina, and facilitating obturation by acting as a lubricant and entrapping the remaining bacteria ${ }^{3}$.

Among the root canal sealers, epoxy resin-based sealers are one of the commonly used dental materials. These sealers have superior adhesion properties with collagen in root dentin compared to mineral trioxide aggregate ${ }^{4)}$, zinc oxide eugenol ${ }^{5)}$, and calcium hydroxide ${ }^{6,7)}$. In addition to conventional materials, bioactive materials have been used in the dental field for their beneficial properties ${ }^{8}$. Incorporation of bioactive particles in conventional sealers can provide the same desired effect of the bioactive materials ${ }^{9)}$. These materials can increase the sealing ability by closely adapting to root dentin ${ }^{10)}$ and can provide a biological response at the material-dentin interface. Moreover, they have an antibacterial effect, which can improve the quality and longevity of RCT ${ }^{111}$.

Bioactive glass (BG) is a silicate-based bioactive material that contains calcium and phosphate ${ }^{12)}$. It gained researchers' attention due to its mechanical properties (which are comparable to natural tissues),

Color figures can be viewed in the online issue, which is available at J-STAGE.

Received Mar 4, 2019: Accepted Jun 12, 2019

doi:10.4012/dmj.2019-064 JOI JST.JSTAGE/dmj/2019-064 its biological effect on the cells by enhancing bone formation, and its ability to be converted into apatitelike material ${ }^{13)}$. Furthermore, fluoride incorporation in $B G$ can result in the formation of fluorapatite (FA), which is more acid resistant than hydroxyapatite (HA) and has an antimicrobial effect against Enterococcus faecalis in root canals ${ }^{14)}$.

Previously, nano-sized, radio-opaque bioactive glass particles were incorporated into AH Plus endodontic sealer. Over the course of 28 days, a sharp increase in the $\mathrm{pH}$ of the BG-loaded sealer was compared to pure AH Plus root canal sealer. The bioactivity showed formation of calcium phosphate crystals and increased micro-hardness ${ }^{15}$. Other studies have reported the setting behavior, solubility, and volumetric changes of BG-based sealers ${ }^{16}$. However, after reviewing the current literature, it appears that no study was found in which the effect of fluoridated BG particles in endodontic sealer was investigated. Moreover, no study was identified that related to sealing ability, which is important prior to the clinical use of the material. Therefore, fluoridated BG nanoparticles (F-nBG) were synthesized using the solgel method. After being incorporated into endodontic sealers, the obtained material was comprehensively analyzed. This study hypothesizes that F-nBG added sealers will enhance the adhesion and fluoride-releasing properties and may overcome sealing drawbacks associated with conventional sealers.

\section{MATERIALS AND METHODS}

The fluoridated BG was synthesized using the sol-gel 
method, as described previously ${ }^{17)}$. The concentration of fluoride in the BG was $5 \mathrm{~mol} \%$. Two types of experimental bioactive sealers were synthesized by incorporating fluoridated BG nano-powders (F-nBG) uniformly in AH Plus $^{\circledR}$ (AH Plus root canal sealer, DENTSPLY DeTery, Konstanz, Germany). The concentration of F-nBG powder was $2.5 \%$ and $5 \% \mathrm{wt} / \mathrm{wt}$ and denoted as $\mathrm{AH}$ FBG2.5 and AH-FBG5, respectively. AH Plus without F-nBG was used as a control material.

\section{Setting time and flowability}

Silicon molds of $5-\mathrm{mm}$ diameter and 2-mm height were used to prepare samples of each group $(n=5)$. The molds were filled with freshly prepared materials to test setting time in accordance with the ISO 6876 specifications. A Gilmore needle apparatus was used with the flat end of diameter $2-\mathrm{mm}$ to assess the setting time of control and experimental sealers at $37^{\circ} \mathrm{C}$ and 95\% humidity using an incubator (IncuSafe, MCO-5AC, SANYO, Sanyo-Onoda, Yamaguchi, Japan) to simulate the oral cavity temperature. Setting time was recorded when indentations on sealer surfaces were not visible. Digital photographs of the sealers in the Gilmore needle were obtained after $30 \mathrm{~min}$ of the initial mix. Each sealer string was measured at five different points using ImageJ software developed by National Institutes of Health to record the extent of flowability.

\section{Fourier transform infrared spectroscopy (FTIR)}

The control and experimental samples with $5 \times 2 \mathrm{~mm}^{2}$ dimensions were fabricated using silicon molds. Each sample was immersed in 7-mL of deionized water and stored at $37^{\circ} \mathrm{C}$ for $24 \mathrm{~h}$. Then FTIR of all samples from each group $(n=5)$ was conducted using the ATR accessory of a Thermo Nicolet 6700 (ThermoFisher Scientific, Waltham, MA, USA). The resolution was $8-\mathrm{cm}^{-1}$ with a scan number of 256 . The spectral range was $4,000-525$ $\mathrm{cm}^{-1}$. OMINIC software was used to conduct the peak height analysis of the obtained spectra.

\section{Fluoride release analysis}

Samples with $5 \times 2 \mathrm{~mm}^{2}$ dimensions were fabricated using silicon molds. After preparing control and experimental sealers $(n=6)$, each sample was immersed in $7-\mathrm{mL}$ of deionized water ( $\mathrm{pH} 7$ ) and stored at $37^{\circ} \mathrm{C}$ for $24 \mathrm{~h}$. For fluoride release analysis, the samples were dried and transferred into fresh media at intervals of $1,3,7,14$, 30 , and 40 days. After, the media were evaluated using inductively coupled plasma optical emission spectroscopy (Avio 500, Perkin Elmer, Waltham, MA, USA) to measure the fluoride release. The mean cumulative fluoride release behavior was determined.

\section{Water absorption and sorption measurements}

After the control and experimental sealers were initially prepared as described as mentioned previously, water absorption and sorption were determined in deionized water. All samples from each group $(n=6)$ were weighed in a pre-calibrated weighing balance (DAB220, WENSAR, Chennai, India), where the initial mass was considered to be $\mathrm{M}_{0}$. Later, after being immersed in deionized water, all samples were analyzed periodically at days $1,3,7$, 14, 30, and 40. After the samples were removed from the media, they were dried on both sides using filter paper and weighed to record their water absorption. The obtained values were denoted as $\mathrm{M}_{1}$. The samples were then stored under dry conditions at $37^{\circ} \mathrm{C}$ and were weighed until the mass equilibrium was achieved. The final mass was recorded as $\mathrm{M}_{2}$. Water sorption (W) was calculated as percentages using the following equation:

$$
\mathrm{W}(\%)=\left(\left(\mathrm{M}_{2}-\mathrm{M}_{0}\right) /\left(\mathrm{M}_{0}\right) \times 100\right)
$$

eq. 1

and for water absorption (A):

$$
\mathrm{A}(\%)=\left(\left(\mathrm{M}_{1}-\mathrm{M}_{0}\right) /\left(\mathrm{M}_{0}\right) \times 100\right)
$$

eq. 2

\section{Sealing ability analysis}

\section{Teeth preparation}

After obtaining ethical permission from the institutional ethical committee (EA: 2017002), human extracted single-rooted teeth were collected from the department of oral surgery. Verbal consent was taken from patients. Intact, non-carious teeth were selected, disinfected, and stored in a saline solution with $1 \%$ thymol at $4^{\circ} \mathrm{C}$ until prepared. The crowns were removed at the cementoenamel junction using a high-speed bur with water spray. Twelve randomly selected roots were prepared after determining working length by subtracting 1-mm from the length of an inserted \#15 K-file (DENTSPLY Maillefer, Ballaigues, Switzerland) with its tip visualized at the apical foramen. The canals were instrumented using a 6\% tapered K3 rotary system (SybronEndo, K3, Kerr, Glendora, CA, USA) with size 40 as a master apical file. Between each file size, 3-mL 2.5\% sodium hypochlorite $(\mathrm{NaOCl})$ was introduced to irrigate the canal. After preparation, 3-mL 5.25\% NaOCl, followed by 5 -mL 18\% EDTA (Ultradent, South Jordan, UT, USA) were used for 60-s to remove the smear layer. All canals were washed thoroughly by deionized water and dried with sterile paper points.

The prepared canal walls $(n=4)$ were coated with the control and experimental sealers by using the master cone i.e. $2 \%$ taper gutta-percha (Sure-endo, 2\%ISO Size, Sure Dent, Sagimakgol-ro, Korea) of size 40. Afterward, size 20 spreader (Tapered Finger spreader, Dentsply, Ballaigues, Switzerland) was used in cold lateral condensation technique to add $2 \%$ taper of size 20 accessory cones. A heated plugger (Hand plugger, Schwert, Seitingen-Oberflacht, Germany) was used to cut the cone at 1-mm level of the roots with a layer of sealer coated on the top to ensure a proper coronal seal. A single operator (Y.K.A) conducted this procedure.

2. Micro-computed tomography (micro-CT) analysis The sealing ability of the groups $(n=4)$ along with the walls of root dentin was determined by measuring the differences in percentage voids volume (voids\%) using a high-resolution micro-CT (SkyScan, 1176, BrukermicroCT, Kontich, Belgium). The teeth were scanned 
periodically — at 0, 1, 30, 60, and 90 days of preparation and root filling- and samples were stored in deionized water at $37^{\circ} \mathrm{C}$. The media were changed after every third day. A trained and calibrated single operator scanned each sample using micro-CT (Skyscan, 1172 machine, Bruker-microCT) with the parameters of source voltage $90-\mathrm{kV}$, source current of $112-\mathrm{uA}$, image pixel size 10.02 $\mu \mathrm{m}$, filter $=\mathrm{Al}+\mathrm{Cu}$, exposure of $1,150-\mathrm{ms}$, rotation step $0.500 \mathrm{deg}$, frame averaging $=2$, random movement $=10$, and $360^{\circ}$ rotation. Raw images with tiff format were reconstructed using NRecon Version: 1.6.4.8, Skyscan 2011 software. The CTan Version 1.11.10.0+(64-bit), Skyscan 2003-11 software was used to calculate the total volume of canal and voids in cubic millimeter using the equation:

Voids\%=((Intended day voids volume-canal volume at baseline $) \times 100$ ) eq. 3

\section{Push-out bond strength}

To evaluate the push-out bond strength, roots were molded in self-cured acrylic and sectioned transversely into 2-mm-thick discs $(n=12)$ using a precise cutting machine with a diamond saw (Isomet, Buehler, Lake Bluff, NY, USA). Afterward, teeth were prepared to be 1.3-mm-diameter, and the inner surfaces of sectioned teeth were washed with $18 \%$ EDTA and $2.5 \% \mathrm{NaOCl}$ and dried using paper points.

The sealers were mixed and packed in each disc as per the protocol described as mentioned previously. After filling, samples were stored in deionized water at $37^{\circ} \mathrm{C}$ and $95 \%$ humidity for periodic time intervals: 7, 30, and 90 days. The push-out test was performed periodically at a crosshead speed of $0.5 \mathrm{~mm} \cdot \mathrm{min}^{-1}$ using a universal testing machine (Instron 8871, Instron, High Wycombe, England). The maximum load at failure $\left(\mathrm{F}_{\max }\right)$ was recorded in $\mathrm{N}$, and the bonding surface (A) was calculated using the equation:

$$
\mathrm{A}=2 \pi r \times h
$$

eq. 4

Where $\Pi$ is the constant 3.14, $r$ is the root canal space radius, and $h$ is the thickness of the slice in $\mathrm{mm}$. Then, the bond strength expressed in $\mathrm{MPa}$.

\section{Statistical analysis}

Statistical analysis was performed using SPSS-22 (IBM SPSS ${ }^{\circledR}$ software, Atlanta, GA, USA). The results were presented in mean $\pm(\mathrm{SD})$. An analysis of variance
(ANOVA) post-hoc Tukey's test was used to evaluate the significance values in relation to a control group with experimental groups into specified days. The level of statistical significance was considered to be less than or equal to the $p$-value of 0.05 .

\section{RESULTS}

\section{Setting time and flowability}

Experimental endodontic sealers (AH-FBG2.5 and AH-FBG5) presented significantly lower initial setting time compared to AH Plus $(p<0.000)$. However, the setting time is within the acceptable range for clinical applications as tabulated in Table 1. Flowability (Fig. 1) decreased when the percentage of F-nBG particles was increased, where a mean length of $18.6 \pm 4 \mathrm{~mm}$ for control was compared (Table 1) to $12.2 \pm 3$ and $9 \pm 4 \mathrm{~mm}$ for AH-FBG2.5 and AH-FBG5, respectively. The width of extended sealers was assessed from three different points, and an average width of each material is given in Table 1, however, the difference was non-significant.

\section{Fourier transform infrared spectroscopy (FTIR)}

Figure 2a shows the comparative spectra of AH Plus, AH-FBG2.5, and AH-FBG5. The spectra show characteristic peaks of $\mathrm{AH}$ Plus, where an $\mathrm{O}-\mathrm{H}$ band appeared at 3,500 to $3,200 \mathrm{~cm}^{-1}$, and an $\mathrm{N}-\mathrm{H}$ peak appeared at $3,028-\mathrm{cm}^{-1}$. AH Plus contains bisphenol A epoxy resin, bisphenol $\mathrm{F}$ epoxy resin in paste $\mathrm{A}$ and dibenzyldiamine, adamantane amine, and tricyclodecane diamine in paste $\mathrm{B}$. The peaks at 1,604 and $1,508 \mathrm{~cm}^{-1}$ were attributed to aromatic groups, whereas the peak at

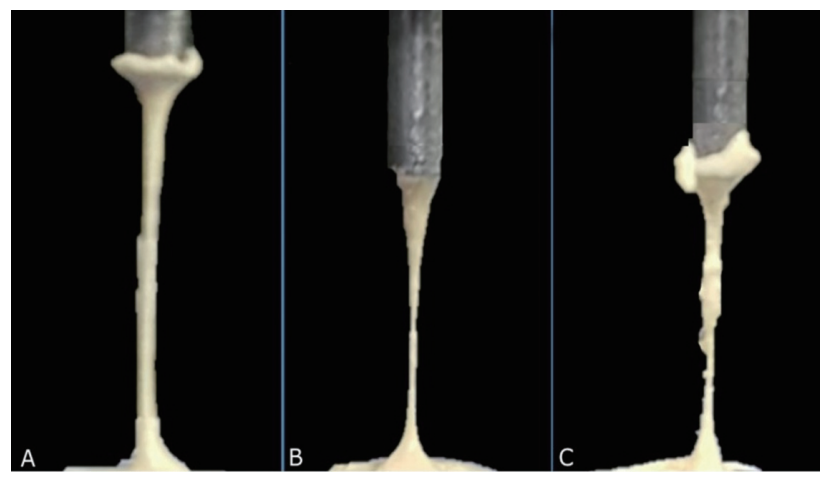

Fig. 1 Flowability of specimens observed after $30 \mathrm{~min},(\mathrm{~A})$ AH Plus, (B) AH-FBG2.5, and (C) AH-FBG5.

Table 1 Comparative setting time and flowability values of AH-FBG2.5 and AH-FBG5 and AH Plus

\begin{tabular}{lccc}
\hline \multirow{2}{*}{ Groups } & Setting time $(\mathrm{min})$ & Flow $(\mathrm{mm})$ & Width $(\mathrm{mm})$ \\
\cline { 2 - 4 } & Mean (SD) & Mean (SD) & Mean (SD) \\
\hline AH Plus & $363.2(3.11)$ & $18.6(4)$ & $4.14(1.7)$ \\
AH-FBG2.5 & $172.8(2.17)$ & $12.2(3)$ & $3.28(2.1)$ \\
AH-FBG5 & $188.6(1.14)$ & $9(4)$ & $4.67(2.2)$ \\
\hline
\end{tabular}



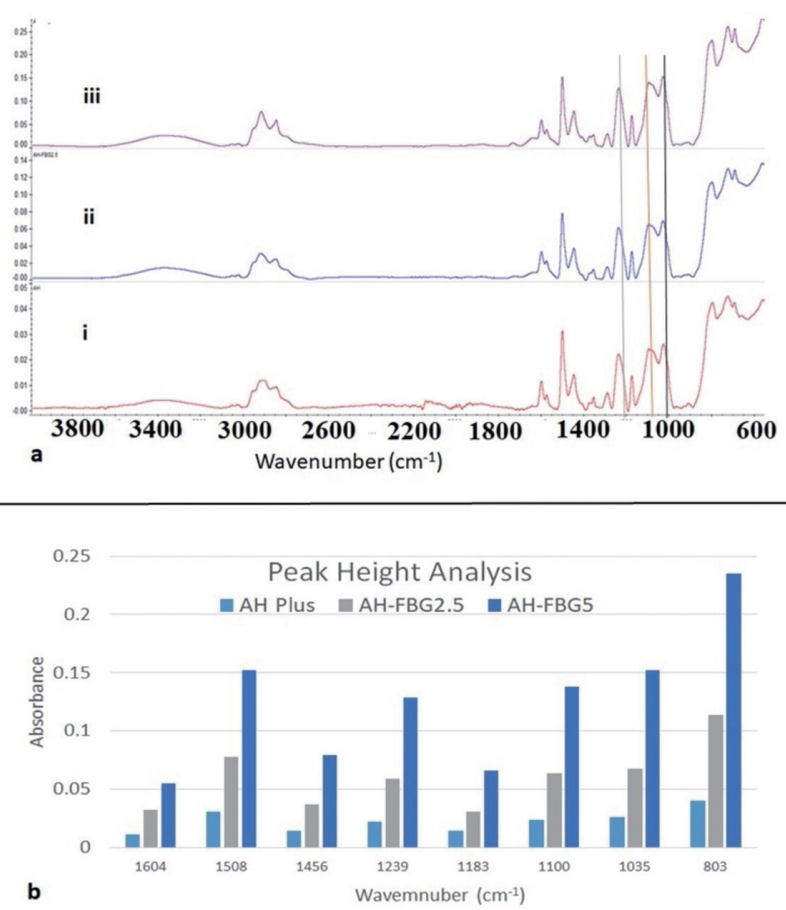

Fig. 2 (a) Comparative FTIR spectra of (i) AH Plus, (ii) AHFBG2.5, and (iii) AH-FBG5, shifting in peaks were observed and (b) change in peak height analysis were observed with increase in the concentration of F-nBG.

1,456- $\mathrm{cm}^{-1}$ corresponded to bending absorbance of $\mathrm{N}-\mathrm{H}$ groups. The stretching vibrating peaks of $\mathrm{C}-\mathrm{N}$ appeared at 1,296 and $1,239-\mathrm{cm}^{-1}$. The peak at $1,183-\mathrm{cm}^{-1}$ was attributed to $\mathrm{Si}-\mathrm{CH}$ due to the presence of silicone oil in AH Plus, while the epoxide band appeared at $1,100-1,030 \mathrm{~cm}^{-1}$. The peak at $803-\mathrm{cm}^{-1}$ was attributed to Si-O. With the inclusion of FBG, the characteristic peaks of BG did not appear separately, as the peak of FBG appears in the same region. However, shifting of peaks was observed at $1,030-\mathrm{cm}^{-1}$ due to the presence of FBG particles. It was also observed that the intensities of peaks increased with the inclusion of FBG in AH-Pus; moreover, the intensities increased with the increase in the concentration of nanoparticles, as shown in Fig. 2 b.

\section{Fluoride release analysis}

The fluoride release behavior of experimental sealers is given in Fig. 3a. It was observed that the release profile was initially higher, where the values at day 1 for AH-FBG2.5 and AH-FBG5 were $0.72 \pm 0.009$ and $0.8 \pm 0.02 \mathrm{mg} / \mathrm{L}$, respectively. However, the maximum fluoride release values were observed at day 7: $\mathrm{AH}-$ FBG2.5 0.79 $\pm 0.006 \mathrm{mg} / \mathrm{L}$ and AH-FBG5 0.90 \pm 0.009 $\mathrm{mg} / \mathrm{L}$. It was found that the release values decreased with a further time interval and had decreased significantly by day 40: $p<0.05$. No fluoride release was observed from the control group. When comparing experimental sealers, AH-FBG5 showed an insignificant difference $(p>0.05)$ in release of fluoride compared with AH-FBG5.
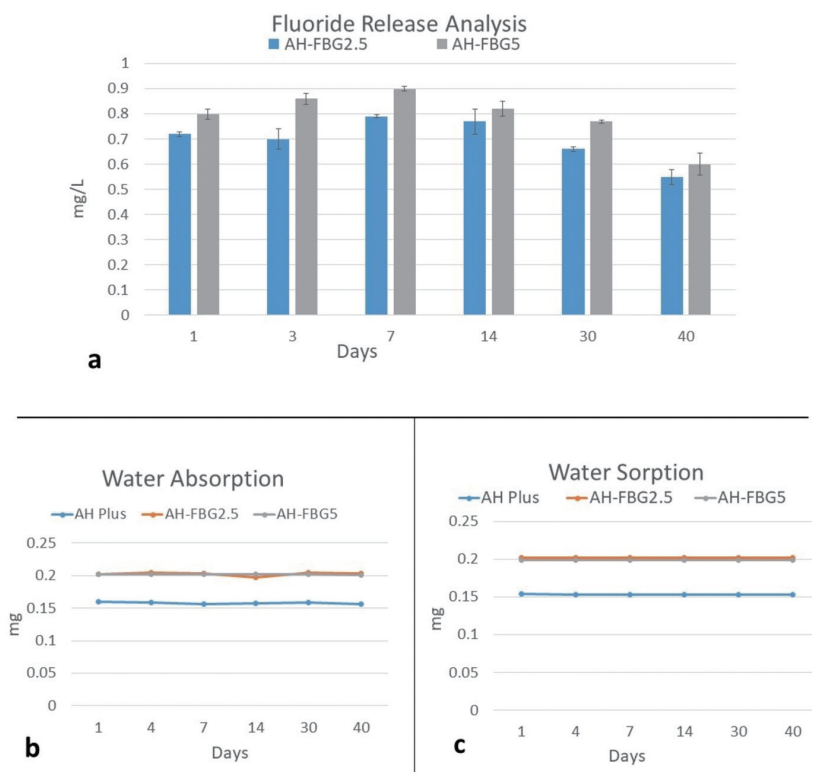

Fig. 3 (a) Fluoride release analysis of AH-FBG2.5 and AHFBG5, (b) Water absorption, and (c) water sorption measurement (weight analysis) with periodic time intervals.

Water absorption and sorption measurements

For water absorption and sorption, samples did not exhibit a significant change in different intervals, as shown in Fig. 3 (b \& c). Statistical similarity for weight change was observed among all groups, with a gradual increase in time for reaching equilibration.

\section{Sealing ability analysis}

The void volume percentage values in the control group were decreased from day 1 to day 90 with non-linear behavior as shown in Table 2. Experimental sealers showed a gradual decrease in voids volume percentage periodically. However, there was a non-significant difference $(p>0.05)$. The voids $\%$ was significantly lower $(p<0.05)$ in the control sealer than the experimental sealer AH-FBG2.5. The mean difference reduction was insignificant within the groups (Table 2). The crosssectional 2D images (Fig. 4i) demonstrated a reduction of voids in alternating scans from day 1 up to day 90; 3D images of root canals are shown in Fig. 4ii.

\section{Push-out bond strength}

The mean values and standard deviations of the pushout bond strength (MPa) at 7, 30, and 90 days are shown in Fig. 4iii. The difference in mean values among groups at all times was significant $(p<0.05)$. The values for the AH Plus sealer were significantly lower than AH-FBG2.5 and AH-FBG5 $(p<0.05)$ at 7,30 , and 90 days; AH-FBG5 had a significantly higher push-out bond strength value than both AH Plus and AH-FBG2.5 $(p=0.0006)$ after 90 days.

The groups showed a significantly higher mean push-out bond strength at 90 days compared to 7 days 
Table 2 Voids (\%) in the sections of the groups at day 7, 30, and 90

\begin{tabular}{lcccccc}
\hline & \multicolumn{5}{c}{ Voids volume [Mean (SD)] } \\
\cline { 2 - 7 } Groups & Day 1 & Day 7 & Day 30 & Day 90 & $\begin{array}{c}\text { Mean difference } \\
(90-1 \text { days })\end{array}$ & $p$ value \\
\hline AH Plus & $1.60(0.59)$ & $1.44(0.45)$ & $1.47(0.45)$ & $1.39(0.49)$ & -0.21 & 0.59 \\
AH-FBG2.5 & $3.60(0.71)^{\mathrm{a}}$ & $3.22(0.50)^{\mathrm{a}}$ & $3.14(0.47)^{\mathrm{a}}$ & $3.05(0.61)^{\mathrm{a}}$ & -0.55 & 0.28 \\
AH-FBG5 & $2.86(0.91)$ & $2.74(0.93)$ & $2.72(0.90)$ & $2.68(0.94)$ & -0.17 & 0.79 \\
\hline
\end{tabular}

a Significant difference of means between control and AH-FBG2.5

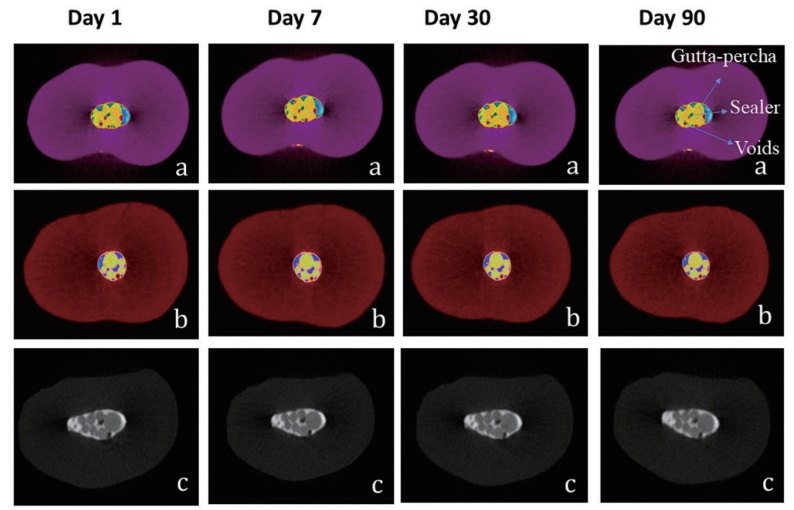

(i)

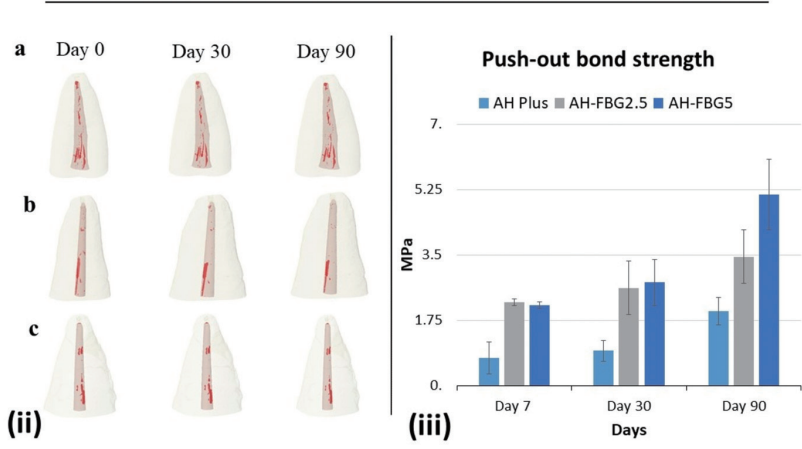

Fig. 4 (i) Representative micro-CT tomography images of (a) AH Plus, (b) AH-FBG2.5, (c) AH-FBG5 taken at days $1,7,30$, and 90 .

The images are color-coded to distinguish the sealer from the gutta-percha. (ii) Representative 3D images of (a) AH Plus, (b) AH-FBG2.5, (c) AHFBG5 taken at days 0,30 , and 90 from same angle/ position, and (iii) Mean push-out bond strength values of control and experimental sealers with periodic time intervals.

for the control $(p=0.018)$ and for AH-FBG2.5 and AHFBG5 ( $p=0.043$ and $p=0.006$, respectively). The mean push-out bond strength values of AH Plus, AH-FBG2.5, and AH-FBG5 at day 7 were $0.73 \pm 0.42,2.23 \pm 0.099$, and $2.15 \pm 0.09 \mathrm{MPa}$, respectively. At day 90 , the values were $1.99 \pm 0.36,3.45 \pm 0.72$, and $5.12 \pm 0.94 \mathrm{MPa}$, respectively.

\section{DISCUSSION}

Recently, there has been a paradigm shift toward the use of bioceramics in restorative materials. This might be attributed to the changing trends in global dental research, which have favored adopting an interdisciplinary approach in order to achieve materials with more optimized clinical properties to perform their desired function. The popularity of bioceramics in dental materials is due to their regenerative potential. In this study, the selected experimental groups (AH-FBG2.5 and AH-FBG5) were determined based on synthesizing an endodontic sealer and exhibiting benefits of F-BG nanoparticles in terms of mechanical, physical, and chemical properties of the material.

One important criterion of an ideal root canal sealer is to have an adequate working time and setting time. Whereby the setting time is mainly a control test to evaluate the stability of material behavior ${ }^{18,19)}$. The incorporation of F-nBG in the experimental sealer samples can contribute to a shorter setting time and deceased flowability when compared to AH Plus sealer. The setting time of the experimental groups was faster and had sufficient flowability after 30-min, which is similar to the average working time for the obturation of single canals found ${ }^{20)}$. Moreover, faster setting can help reducing recontamination possibility.

Adequate flowability can influence the proper penetration of endodontic sealer into irregularities of the dentin, isthmus, ramifications, and accessory canals. Thus, it increases the possibility of attaining a sufficient seal of the root canal system and minimizes voids between master and accessory cones ${ }^{21)}$. On the other hand, reduction in flowability can result in an increased chance of creating voids ${ }^{22}$. However, an excessive increase in sealer flowability may lead to an increased possibility of apical extrusion ${ }^{23)}$. The results of this study showed that AH Plus has the highest flowability; for the experimental sealers, the flowability decreased as the percentage of the particles increased. AH-FBG2.5 showed lesser flowability than AH Plus due to particles incorporation; however, it had an adequate flowability after 30-min of the initial mix.

Sealers solubility testing was measured by the difference in the material mass before and after immersion in deionized water. Particles of the sealer 
material may leach out during storage in liquid. Under the conditions of the present study, no weight change was observed among the groups. This also fulfills the requirement of endodontic sealers with less than $3 \%$ weight loss according to ISO 6876 specifications $^{24)}$.

Fluoride release is one of the major advantages of restorative materials due to its ability to prevent recurrent caries. The study showed an initial burst of fluoride release that was decreased afterward, while the control sealer showed no release of fluoride. The release might stop after a longer period; however, initial fluoride release would greatly benefit $\mathrm{RCT}$ remineralization and could have an antimicrobial effect against entrapped bacteria.

Maximum release was observed at day 7 , which was attributed to the resistance of fluoride ions to move within the resin matrix ${ }^{25}$. The release of fluoride also depends on the interaction of water and change in its $\mathrm{pH}$ due to the exposure of storage media to the external environment and absorption of carbon dioxide from air in addition to temperature change ${ }^{26)}$. In this study, deionized water was the storage media, which has been shown to lead to a higher release of fluoride compared to artificial saliva $^{27)}$.

Presence of voids, particularly in the apical third, can affect the success of the treatment ${ }^{28)}$. Results of the present study show voids\% changes over 90 days within the groups. Experimental groups showed a gradual decrease; the results were consistent with the solubility of materials as volumetric changes can be assessed by solubility and dimensional changes can be assessed by micro-CT ${ }^{29}$. Multiple factors can lead to the presence of voids including changes in sealer manipulation such as manual mixing and carrying, which can lead to air entrapment. In addition, flowability and initial setting time can directly affect void volume ${ }^{30)}$. It is anticipated that the addition of fillers in AH Plus sealer increase voids $\%$ and can decrease flowability, which may inversely affect the sealing ability. However, in this study, voids\% reduction was observed with the experimental selaers, whereby, the biological activity of F-nBG may enhance the formation of an apatite layer, thus, improving the sealing ability of the material ${ }^{31}$.

Adhesion of root canal sealers can result in the greater sealing ability of the material, thus leading to decreased apical and coronal leakage. The adhesion of sealers can be affected by multiple factors including its chemical structure, root site, interaction with root dentin, and presence of a smear layer ${ }^{32}$. Push-out bond strength results in the present study showed that experimental sealers have a higher bond strength than AH Plus sealer. This could be attributed to the material's bioactivity: by forming an apatite layer and depositing hydroxyapatite that chemically bonds to tooth structure.

\section{CONCLUSION}

In conclusion, incorporating F-nBG in AH Plus endodontic sealer resulted in a shorter setting time, relative insolubility, fluoride-releasing ability, and better interfacial adhesion. However, the setting time and flowability of experimental sealers are within the acceptable range for the clinical application. An additional factor of fluoride and BG can help to reduce bacterial contamination. Formation of a new apatite layer with BG can help form a better seal. The results showed that the experimental sealers had better bond strength than the control group. Future studies can be conducted to evaluate the physical and mechanical properties of bioactive endodontic sealers further.

\section{ACKNOWLEDGMENTS}

The authors would like to acknowledge the College of Dentistry, Imam Abdulrahman Bin Faisal University (IAU) for providing partial funding (Grant \# 201807) for this research study. We would like to thank Mr. Lindsey Mateo and Mr. Jim for their expert advice in the laboratory and would like to show our gratitude to the Environmental Engineering Department, IAU, for their help.

\section{REFERENCES}

1) Bansode PV, Pathak SD, Wavdhane MB, Kale D. Obturating materials present and past: A review. IOSR J Dent Med Sci 2018; 17: 27-33.

2) Guimarães BM, Amoroso-Silva PA, Alcalde MP, Marciano MA, de Andrade FB, Durate MA. Influence of ultrasonic activation of 4 root canal sealers on the filling quality. $J$ Endod 2014; 40: 964-968.

3) Kaur A, Shah N, Logani A, Mishra N. Biotoxicity of commonly used root canal sealers: A meta-analysis. J Conserv Dent 2015; 18: 83-88.

4) Silva EJ, Carvalho NK, Prado MC, Zanon M, Senna PM, Souza EM, et al. Push-out bond strength of injectable pozzolanbased root canal sealer. J Endod 2016; 42: 1656-1659.

5) Gurgel-Filho ED, Leite FM, Lima JBD, Montenegro JPC, Saavedra F, Silva EJNL. Comparative evaluation of push-out bond strength of a MTA-based root canal sealer. Braz J Oral Sci 2014; 13: 114-117.

6) Vasconcelos BCD, Bernardes RA, Duarte MAH, Bramante CM, Moraes IGD. Apical sealing of root canal fillings performed with five different endodontic sealers: analysis by fluid filtration. J Appl Oral Sci 2011; 19: 324-328.

7) Rosa RA, Bier CAS, Só MVR, Tanomaru JMG, TanomaruFilho M. Push-out bond strength of calcium hydroxide and mineral trioxide aggregate based sealers to root canal dentin. Rev Odonto Ciênc 2012; 27: 320-324.

8) Khalid MD, Khurshid Z, Zafar MS, Farooq I, Khan RS, Najmi A. Bioactive glasses and their applications in dentistry. J Pak Dent Assoc 2017; 26: 32-38.

9) Khan AS, Syed MR. A review of bioceramis-based dental restorative materials. Dent Mater J 2019; 38: 163-176.

10) Yoshii S, Washio A, Morotomi T, Kitamura C. Root canal sealing ability of bioactive glass-based sealer and its effects on dentin. Jpn J Conserve Dent 2016; 59: 463-471.

11) Malarvizhi D, Kavitha M, Loganathan SC, Karthick A. Evaluation of microleakage of bioactive glass, bioactive glasshydroxyapatite and mineral trioxide aggregate - An in vitro study. World J Med Sci 2014; 10: 208-211.

12) Farooq I, Imran Z, Farooq U, Leghari A, Ali H. Bioactive glass: a material for the future. World J Dent 2012; 3: 199201.

13) Rahaman MN, Day DE, Bal BS, Fu Q, Jung SB, Bonewald LF, et al. Bioactive glass in tissue engineering. Acta Biomater 
2011; 7: 2355-2373.

14) Mathew VB, Madhusudhana K, Sivakumar N, Venugopal T, Reddy RK. Anti-microbial efficiency of silver diamine fluoride as an endodontic medicament - An ex vivo study. Contemp Clin Dent 2012; 3: 262-264

15) Heid S, Stoessel PR, Tauböck TT, Stark WJ, Zehnder M, Mohn D. Incorporation of particulate bioactive glasses into a dental root canal sealer. Biomed Glasses 2016; 2: 29-38.

16) Tanomaru-Filho M, Torres FF, Chávez-Andrade GM, de Almeida M, Navarro LG, Steier L, et al. Physicochemical properties and volumetric change of silicone/bioactive glass and calcium silicate-based endodontic sealers. J Endod 2017; 43: 2097-2101.

17) Gul H, Zahid S, Zahid S, Zahid SH, Kaleem M, Khan A, et al. Sol-gel derived fluoride-doped bioactive glass powders: Structural and long-term fluoride release/pH analysis. J NonCryst Solids 2018; 408: 216-222.

18) Al-Haddad A, Ab Aziz C, Zeti A. Bioceramic-based root canal sealers: a review. Int J Biomater 2016; 2016: 9753210.

19) Marín-Bauza GA, Silva-Sousa YT, Cunha SA, Rached-Junior FJ, Bonetti-Filoh I, Sousa-Neto MD, et al. Physicochemical properties of endodontic sealers of different bases. J Appl Oral Sci 2012; 20: 455-461.

20) Wong AW, Zhu X, Zhang S, Li SK, Zhang C, Chu CH. Treatment time for non-surgical endodontic therapy with or without a magnifying loupe. BMC Oral health 2015; 15: 40.

21) Faria-Júnior NB, Massi S, Croti HR, Gutierrez JC, Dametto FR, Vas LG. Comparative assessment of the flow rate of root canal sealers. Rev Odonto Ciênc 2010; 25: 170-173.

22) Hosoya N, Kurayama H, Iino F, Arai T. Effects of calcium hydroxide on physical and sealing properties of canal sealers. Int Endod J 2004; 37: 178-184.

23) McMichen FR, Pearson G, Rahbaran S, Gulabivala K. A comparative study of selected physical properties of five rootcanal sealers. Int Endod J 2003; 36: 629-635.

24) Dental Root Canal Sealing Materials (ISO 6876). British Standards Institution 2002.

25) Naoum S, Ellakwa A, Martin F, Swain M. Fluoride release, recharge and mechanical property stability of various fluoridecontaining resin composites. Oper Dent 2011; 36: 422-432.

26) Becking LB, Kaplan IR, Moore D. Limits of the natural environment in terms of $\mathrm{pH}$ and oxidation-reduction potentials. J Geol 1960; 68: 243-284.

27) Poggio C, Andenna G, Ceci M, Beltrami R, Colombo M, Cucca L. Fluoride release and uptake abilities of different fissure sealants. J Clin Exp Dent 2016; 8: e284-e289.

28) Kumar A, Pai AV. Comparative assessment of the area of sealer voids in single cone obturation done with mineral trioxide aggregate, epoxy resin, and zinc-oxide eugenol based sealers. Saudi Endod J 2016; 6: 61-65.

29) Silva EJ, Perez R, Valentim RM, Belladonna FG, De-Deus GA, Lima IC, et al. Dissolution, dislocation and dimensional changes of endodontic sealers after a solubility challenge: a micro-CT approach. Int Endod J 2017; 50: 407-414.

30) Akman M, Akman S, Derinbay O, Belli S. Evaluation of gaps or voids occurring in roots filled with three different sealers. Eur J Dent 2010; 4: 101-109.

31) Viapiana R, Moinzadeh AT, Camilleri L, Wesselink PR, Tanomaru Filho M, Camilleri J. Porosity and sealing ability of root fillings with gutta-percha and BioRoot RCS or AH Plus sealers. Evaluation by three ex vivo methods. Int Endod J 2015; 49: 774-782.

32) Barbizam JV, Trope M, Tanomaru-Filho M, Teixeira EC, Teixeira FB. Bond strength of different endodontic sealers to dentin: push-out test. J Appl Oral Sci 2011; 19: 644-647. 